\title{
Mamoplastía de reducción: resultados desde la perspectiva del paciente. Validación lingüística y psicométrica del Breast $Q$ Reduction and Mastopexy Module Instrument*
}

\author{
Drs. PEDRO CUEVAS T. ${ }^{1}$, MARÍA E. CALDERÓN G. ${ }^{1}$, CRISTIAN ERAZO C. ${ }^{1}$, \\ SUSANA BENÍTEZ S. ${ }^{1}$, PATRICIO ANDRADES C. ${ }^{1}$, SERGIO SEPÚLVEDA P. ${ }^{1,2}$, \\ Alumnos JOSÉ IGNACIO VERGARA ${ }^{3}$, FELIPE SOTO ${ }^{3}$, ALMA CRUZ $^{3}$, Dr. STEFAN DANILLA E. ${ }^{1,2}$
}

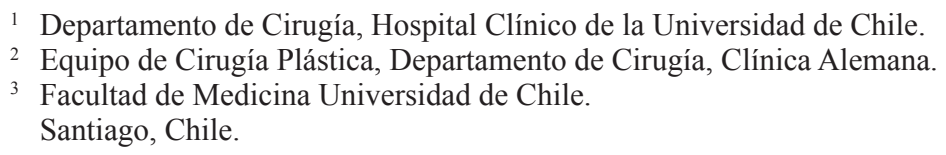

Abstract

\section{Validation of the Spanish version of the breast $Q$ reduction and mastopexy module}

Background: The Breast Q Reduction and Mastopexy Module evaluate breast related quality of life from the perspective of the patients. Body self-image, social performance, sexuality and physical symptoms are assessed. Aim: To translate the Breast Q Reduction and Mastopexy Module to Spanish and validate the translated version to be used in Chile. Material and Methods: The linguistic validation guidelines of the MAPI/TRUST Research institute were used and the survey was applied to six patients aged $29 \pm 6$ years with $14 \pm 2$ years of studies. The internal stability of the test was assessed using Cronbach alpha. Results: The mean body mass index of the patients was $25 \pm 1 \mathrm{~kg} / \mathrm{m}^{2}$. The internal stability of the scale was $97 \%$. The scores of the test in the preoperative period ranged from 74 to 125 points. Conclusions: The Breast Q Reduction and Mastopexy Module is a valid instrument to assess the results of reductive mammoplasty among Spanish speaking patients.

Key words: Mammoplasty, quality of life, Breast Q Reduction and Mastopexy Module.

\section{Resumen}

Objetivo: El Breast Q Reduction and Mastopexy Module es un instrumento específico para la evaluación de calidad de vida asociado a mamas desde la perspectiva del paciente, publicado originalmente en inglés, el cual cuenta con una evaluación pre y otra postoperatoria. El objetivo del presente estudio es traducir

*Recibido el 12 de agosto de 2012 y aceptado para publicación el 5 de noviembre de 2012.

Los autores no refieren conflictos de interés.

Correspondencia: Dr. Stefan Danilla E. Santos Dumont 999, Santiago, Chile. drstefandanilla@gmail.com 
y validar este instrumento al español para su uso en Chile. Material y Método: Se utilizaron las guías de validación lingüística del MAPI/TRUST Research institute. El proceso de validación requirió la traducción ingles-español, contra traducción español-inglés, conciliación inglés-inglés y aplicación piloto de la escala en al menos 5 sujetos. La estabilidad interna se evaluó con el alfa de Cronbach, se utilizó estadística descriptiva y analítica para analizar los resultados. Resultados: Las 6 pacientes evaluadas tuvieron una edad promedio de $29 \pm 16(\mathrm{SD})$ años, IMC $25 \pm 1 \mathrm{~kg} / \mathrm{m}^{2}$ y $14 \pm 2$ años de estudio. La estabilidad interna de la escala fue de $97 \%$. Los resultados obtenidos entre el preoperatorio de las pacientes, variaron de 74 a 125 puntos, con una mediana de 98 puntos. Conclusiones: El instrumento Breast Q Reduction and Mastopexy Module es un instrumento válido, confiable y reproducible para la evaluación de los resultados de la mamoplastía de reducción, desde la perspectiva de los pacientes chilenos hispanoparlantes. Module.

Palabras clave: Mamoplastía de reducción, evaluación, resultados, Breast Q Reduction and Mastopexy

\section{Introducción}

Los resultados en cirugía plástica reconstructiva y estética (CIPRES) abarcan los más diversos ámbitos en la vida de la persona, incluyendo aspectos que tradicionalmente no han sido evaluados por los cirujanos. En la actualidad existe claridad respecto a que la mera evaluación de aspectos técnicos y funcionales resulta insuficiente y no tiene correlación necesariamente con las expectativas de los pacientes. Debido a lo anterior existe una tendencia creciente a incluir en la evaluación de los resultados la perspectiva del paciente, a fin de que esta evaluación sea lo más integral posible. Dicha tendencia se ve reflejada en la aparición de instrumentos de evaluación que incorporan como elemento esencial, la evaluación de resultados desde la perspectiva del paciente. El poder entregar un resultado que cumpla con las expectativas del paciente, representa un desafío para el cirujano; no sólo por la destreza o habilidades quirúrgicas necesarias, si no porque la visión del médico tratante puede no coincidir con la del paciente. En el año 2009, Pusic y cols., publicaron el Breast $Q$ Reduction and Mastopexy Module, estudio en el que se aplicó una encuesta de cuatro dominios, dividida en treinta y nueve ítems logrando cuantificar el grado de satisfacción de los pacientes con su autoimagen corporal, desempeño social, sexualidad y síntomas físicos en relación a sus mamas, aplicando éste instrumento en el pre y postoperatorio de los pacientes que fueron sometidos a una mamoplastía de reducción. Los resultados obtenidos por los creadores han sido replicados por otros cirujanos, con una adecuada consistencia interna y validez de constructo, similar al estudio original. Por lo tanto, decidimos emplear el instrumento en nuestros pacientes y evaluar si obteníamos resultados reproducibles, logrando objetivar con un puntaje, el nivel de agrado o desagrado frente a los resultados de una mamoplastía reductiva. Sin embargo, para la correcta aplicación de un instrumento realizado originalmente en otro idioma, éste debe ser traducido y validado en el español local, de forma que mantenga el mismo grado de consistencia interna y conservar el rigor científico en su aplicación.

El objetivo del presente estudio fue traducir y validar el Breast Q Reduction and Mastopexy Module al español chileno.

\section{Material y Método}

\section{Diseño}

Para lograr nuestro objetivo realizamos un estudio de validación lingüística y adaptación cultural, basados en las guías de validación lingüística MAPI/ TRUST Research institute ${ }^{3}$.

El proceso de validación consta de los siguientes pasos: la traducción del instrumento de inglés a español, contra-traducción español a inglés, conciliación inglés-inglés y aplicación de la encuesta en 6 individuos, algoritmo que se muestra en la Figura 1.

\section{Instrumento de medición}

La encuesta original en inglés incluye 4 dominios divididos en 39 ítems pertenecientes a dominios autoimagen corporal, desempeño social, sexualidad y síntomas físicos; administrados en preguntas tipo Likert de 5 puntos (Tabla 1).

Las propiedades psicométricas de la escala en su publicación original fueron: estabilidad interna evaluada por alfa de Cronbach $>0,85$; correlación inter ítems medida por test re test $>0,70^{1}$.

La escala tipo Likert o evaluación sumaria ${ }^{4}$, es un tipo de escala psicométrica, en la que se especifica el nivel de acuerdo o desacuerdo con una declaración (elemento, ítem o pregunta).

\section{Estadística}

Los resultados se presentan resumidos con estadística descriptiva utilizando promedio \pm desviación estándar para las variables continuas, mediana para las ordinales y frecuencia y porcentaje para las categóricas. La estabilidad interna de la escala se midió 


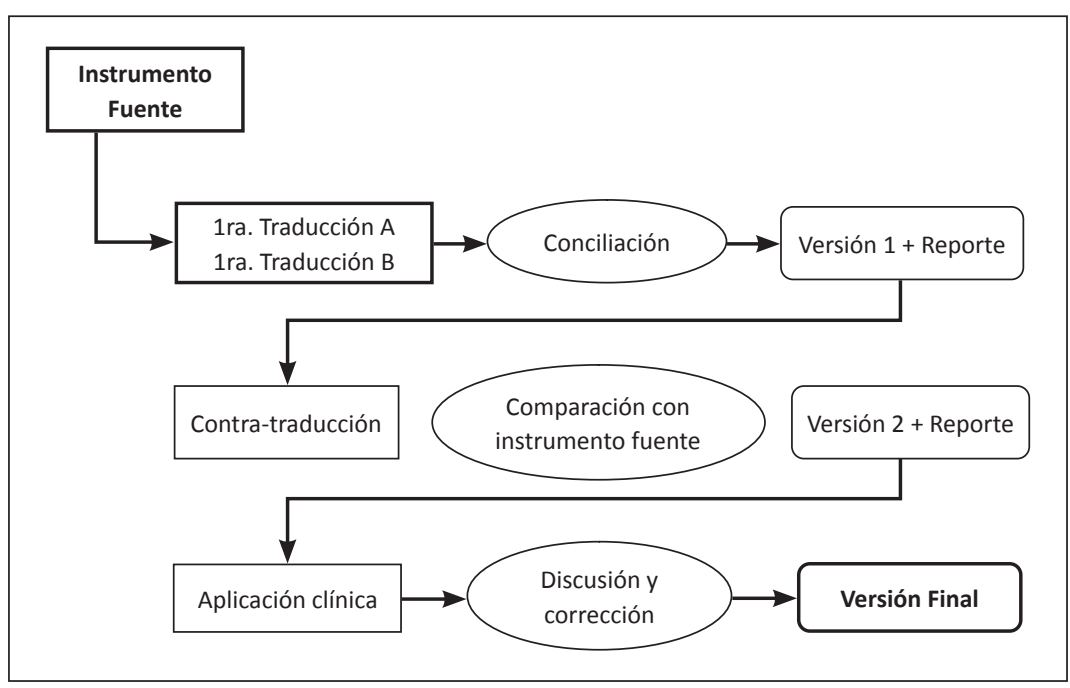

Figura 1. Algoritmo del proceso de validación Lingüística según la MAPI/TRUST Research institute.
Tabla 1. Dominios para evaluación de resultados en Mamoplastía de reducción:

Breast $Q$ Reduction and Mastopexy Module

\begin{tabular}{|c|c|}
\hline 1. Autoimagen corporal & 11 ítems \\
\hline 2. Desempeño social & 9 ítems \\
\hline 3. Sexualidad & 5 ítems \\
\hline 4. Síntomas físicos & 14 ítems \\
\hline
\end{tabular}

con el alfa de Cronbach. La comparación antesdespués del puntaje de la escala se realizó con el test de Wilcoxon para muestras pareadas considerando significativo un valor alfa de 0,0500 (Stata $10 \cdot 2 \AA$ ).

\section{Resultados}

Luego de la traducción, contratraducción y conciliación, se contó con un instrumento de 4 dominios y 39 ítems el que se presenta en la Tabla 2. El ins- trumento fue aplicado a 6 pacientes agendadas para mamoplastía reductiva (Tabla 3 ). La estabilidad interna medida por el alfa de Cronbach fue de $97 \%$.

Las pacientes encuestadas tuvieron una edad de $29 \pm 16$ años, IMC $25 \pm 1 \mathrm{~kg} / \mathrm{m}^{2}$ y $14 \pm 2$ años de estudio (Tabla 3). En la Tabla 4 se puede observar el porcentaje del máximo teórico de puntaje por dominio en el pre y postoperatorio. Se observa que en todos los dominios evaluados hubo mejoría, alcanzando esta misma, un valor estadísticamente significativo para el dominio de síntomas físicos. En el dominio de sexualidad se aprecia la menor variación, pero se debe considerar que sólo 4 pacientes de las 6 evaluadas respondieron ese dominio, las restantes no lo respondieron dado su inactividad sexual. No hubo problemas en la comprensión de las preguntas por ninguno de los pacientes encuestados.

\section{Conclusiones}

El poder evaluar el grado de satisfacción de una cirugía por parte del paciente está adquiriendo cada

Tabla 2. Dominio sexualidad

\begin{tabular}{|lcccccc|}
\hline & Nunca & $\begin{array}{c}\text { Poca } \\
\text { parte del } \\
\text { tiempo }\end{array}$ & $\begin{array}{c}\text { Parte } \\
\text { del } \\
\text { tiempo }\end{array}$ & $\begin{array}{c}\text { La } \\
\text { mayoría } \\
\text { del tiempo }\end{array}$ & $\begin{array}{c}\text { Todo } \\
\text { el } \\
\text { tiempo }\end{array}$ & $\begin{array}{c}\text { No co- } \\
\text { rresponde }\end{array}$ \\
$\begin{array}{l}\text { a. ¿Cómoda/relajada durante las relaciones sexuales? } \\
\text { b. ¿Segura sexualmente? }\end{array}$ & 1 & 2 & 3 & 4 & 5 & $\mathrm{~N} / \mathrm{C}$ \\
c. ¿Satisfecha con su vida sexual? & 1 & 2 & 3 & 4 & 5 & $\mathrm{~N} / \mathrm{C}$ \\
d. ¿Sexualmente atractiva estando vestida? & 1 & 2 & 3 & 4 & 5 & $\mathrm{~N} / \mathrm{C}$ \\
e. ¿Sexy estando desnuda? & 1 & 2 & 3 & 4 & 5 & $\mathrm{~N} / \mathrm{C}$ \\
\hline
\end{tabular}


Tabla 3. Características generales paciente sometidas a mamoplastía reductiva

\begin{tabular}{|ccclcc|}
\hline Paciente & Edad & IMC & Pedículo & Resección & Promedio \\
1 & 17 & 25,4 & Superomedial & 760 & \\
2 & 20 & 25,7 & Superior & 355 & \\
3 & 49 & 24,1 & Superomedial & 150 \\
4 & 22 & 25,2 & Superior & 490 \\
\hline 5 & 16 & 25,3 & Superomedial & 150 \\
\hline 6 & 50 & 25 & Superomedial & 300 \\
\hline
\end{tabular}

Tabla 4. Porcentaje del máximo teórico pre y postoperatorio

\begin{tabular}{|lccc|}
\hline Dominio & $\begin{array}{c}\text { Evaluación } \\
\text { preoperatoria }\end{array}$ & $\begin{array}{c}\text { Evaluación } \\
\text { postopera- } \\
\text { toria }\end{array}$ & p value \\
\hline Autoimagen corporal & $35,6 \%$ & $60,5 \%$ & 0,2366 \\
Desempeño social & $44,8 \%$ & $62,9 \%$ & 0,3309 \\
Sexualidad & $36 \%$ & $41,3 \%$ & 0,7040 \\
Síntomas físicos & $33 \%$ & $80 \%$ & 0,0056 \\
\hline
\end{tabular}

vez una mayor trascendencia, tanto del punto de vista científico al permitir objetivar y cuantificar los resultados con una u otra técnica quirúrgica, evaluar una curva de aprendizaje o estudios epidemiológicos; como también desde el punto de vista de la relación médico-paciente, al poder demostrarle los cambios y hacerlo a él partícipe de su propia mejoría 5 .

En cirugía reconstructiva, resulta muy relevante el poder "cuantificar" los cambios obtenidos entre el pre y el posoperatorio nos puede ayudar a reforzar la sensación de mejoría en nuestros pacientes 6 .

La traducción y validación del instrumento BREAST Q Reduction and Mastopexy Module en "español-chileno" fue realizada para su aplicación a nivel local; sin embargo, la "neutralidad" de la traducción, permitiría su aplicación a nivel del cualquier grupo de pacientes hispanoparlantes, ya que se puso especial énfasis en no usar modismos o "chilenismos".

Si bien en el presente estudio sólo se encontraron diferencias estadísticamente significativas en el dominio de síntomas físicos, creemos que esto obedece al tamaño de la muestra estudiada, ya que no fue el objetivo de este estudio demostrar la capacidad de cambio del instrumento, si no que realizar la validación linguística. Todos los dominios demostraron mejo- ras sustantivas en las evaluaciones postoperatorias, excepto el dominio referido a sexualidad. Sin embargo, cabe mencionar que este dominio no fue respondido por todas las pacientes intervenidas ya que 2 de ellas no han iniciado su actividad sexual.

Con este estudio, se divulga por primera vez un instrumento válido para la evaluación de resultados en la reducción mamaria desde la perspectiva del paciente. Esperamos que su uso se torne rutinario en la evaluación de los pacientes sometidos a mamoplastía reductiva, ya que su uso adecuado permitirá la comparación de resultados entre pacientes y técnicas; permitiendo la realización de estudios de evaluación de resultado y estudios comparativos que sin lugar a dudas contribuirán al avance científico en cirugía plástica.

\section{Referencias}

1. Pusic A, Klassen A, Scott A, Klok J, Cordeiro P, Cano S. Development of a new patient reported outcome measure for breast surgery: The BREAST-Q. Plast Reconstr Surg. 2009;124:345-53.

2. Patel KM, Hannan CM, Gatti ME, Nahabedian MY. A head to-head comparison of quality of life and aesthetic outcomes following immediate, staged-immediate, and delayed oncoplastic reduction mammaplasty. Plast Reconstr Surg. 2011;127:2167-75.

3. Mapi Research Institute. Linguistic Validation of a Patient Reported Outcomes Measure. Lyon-France. 2005.

4. Likert R. A Technique for the Measurement of Attitudes". Archives of Psychology 1932; 140:44-53.

5. Faria F, Guthrie E, Bradbury E, Brain AN. Psychosocial outcome and patient satisfaction following breast reduction surgery. $\mathrm{Br}$ J Plast Surg. 1999;52:448-52.

6. Cano SJ, Browne JP, Lamping DL. Patientbased measures of outcome in plastic surgery: Current approaches and future directions. Br J Plast Surg. 2004;57:1-11. 